\title{
The development of physico-chemico enhanced washing for fine-grained soil
}

\author{
H. Shin \& J. Park \\ Department of Civil and Environmental Engineering, \\ Seoul National University, Korea
}

\begin{abstract}
Soil contamination problems are frequently found in many brownfield redevelopment sites. Various soil remediation technologies have been considered for the clean-up of those contaminated urban renewal sites. Soil washing remediation techniques have been applied, however, granular soils have been treated with soil washing processes since the removal efficiencies on fine-grained soil were very low in most cases. Fine-grained soil, which is considered as waste, has been disposed of at landfills. In this study, novel soil-washing equipment for fine-grained soil, using highly pressurized water to apply physical impact on the slurry and using chemical agents to promote chemical reactions, was developed. Field soil contaminated by heavy metals from an old refinery site in South Korea was investigated to evaluate the contaminant removal efficiency. Ethylene-diamine-tetraacetic acid (EDTA), a chelating agent, was selected to desorb heavy metals from the soil surface. Physical energy was applied using highly pressurized water. Three different kinds of soil-washing methods, including physical desorption, chemical solubilization, and the combined effort, were conducted in this study. The best removal efficiency was found in the combination of physical and chemical treatments at $89 \%$ of $\mathrm{Pb}, 87 \%$ of $\mathrm{Cu}$ and $84 \%$ of As. The test results show that the efficiency in the removal of contaminants in the soil washing system can be enhanced using a physical forcing system.

Keywords: soil washing, fine-grained soil, physical impact force, surfactant, EDTA.
\end{abstract}




\section{Introduction}

Soil contamination problems are frequently found in many construction sites. In urban renewal, the redevelopment of used land, including within old towns, national industrial complexes, train repair areas, army bases and oil and chemical storage buildings is needed nowadays. Those sites have a potential for soil contamination by petroleum and/or heavy metals. If a site is contaminated, then it is supposed to be cleaned up before redevelopment construction. The development of a local community can be delayed, depending on the clean-up technology, and this can cause a loss of value to the site. For this reason, fast and accurate remediation techniques should be selected, with the consideration of the conditions given. In the case of dredging, specifically for the Four Major Rivers Project in South Korea, some of the dredged sediments are contaminated by heavy metals. Contaminated dredged sediments should be treated before their beneficial use in the future. However, the lack of yard for an excessive amount of dredged material and the workability of the treatment, including the remediation technique, should be fast and simple.

Among a number of remediation techniques, such as soil vapor extraction, soil washing, thermal desorption, bioremediation and so on, soil washing has the advantages of a quick treatment period, easy operation and management and the permanent removal of contaminants from the soil with quality control. Furthermore, soil washing can be successfully applicable for combined polluted sites, by controlling the composition of washing agents. In the case of soil washing for fine-grained soil, however, the efficiency of contaminant removal is very low. Thus the fractions of silt and clay after a soil washing process are generally dumped and disposed of in a landfill site. High disposal costs and the limitations of the landfill site in Korea are serious problems in using a conventional soil-washing system.

In this paper, a novel pilot-scale soil washing methodology for fine-grained soil was introduced and evaluated with field contaminated soil to look into the efficiency of the developed equipment.

\section{The novel soil washing system}

The problem with fine-grained soil washing comes from the tendency of contaminants to bind readily to silt, clay and organic matters by physico-chemical interactions. Generally the contaminant concentration of coarse grained soil is much lower than that of the fine grained soil, as the coarse-grained soils show no significant interactions with contaminants. The formation of micro-structural units of fine-grained particles like clusters, pads, particle packets and aggregates also prevent successful washing of the contaminants with the existence of entrapped pores and apparently small specific surface areas of the target soil. In the novel soil washing equipment, highly pressurized water was introduced to solve the problem which had occurred in the 
conventional system. The method comprises of slurry transfer and a mixing process, with applying an impact force process with a solid/liquid separation process. A schematic diagram of the novel soil washing system is shown in Figure 1.

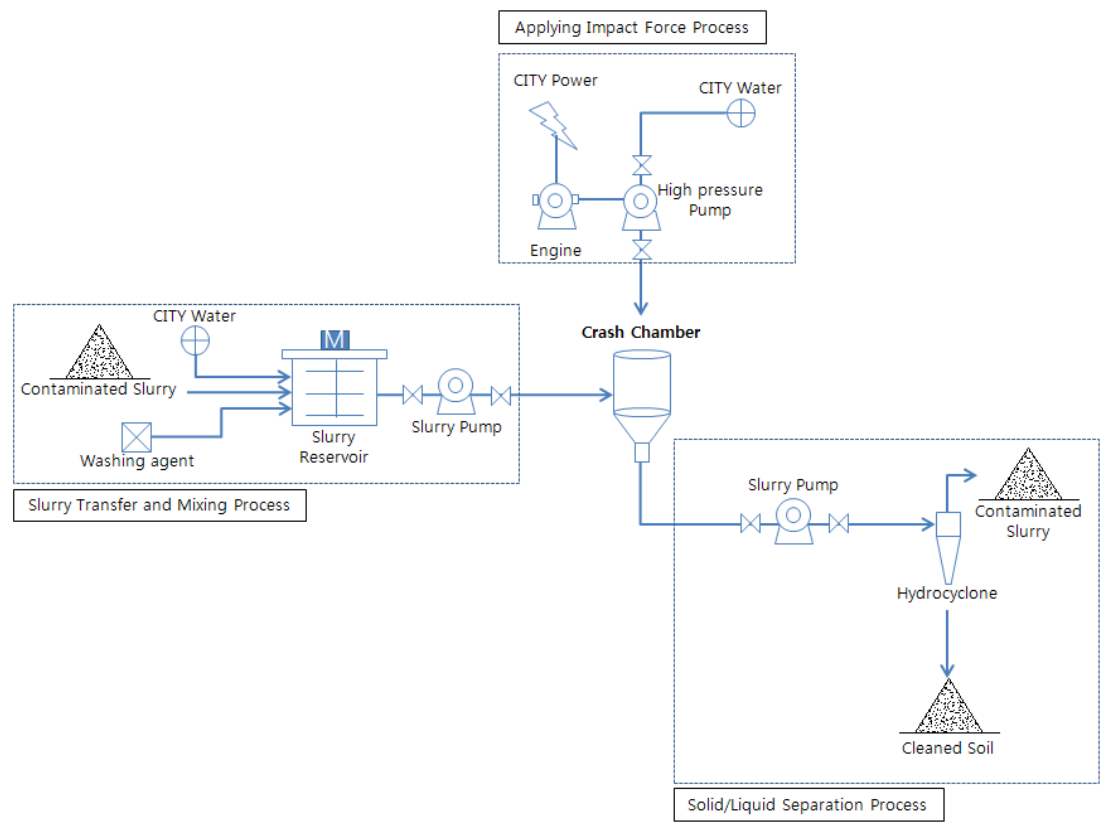

Figure 1: Schematic diagram of novel soil washing system.

In the slurry transfer and mixing process, the contaminated slurry and tap water are mixed to make slurry with a proper soil/water ratio. EDTA for heavy-metal-contaminated soil was added to enhance the removal efficiency. The slurry is well mixed for five minutes and transferred to the crash chamber by a slurry pump. In applying the impact force process, the city water was delivered to create high water pressure using a pump (Model: A10VSO45DFR, Rexroth pump). The designed output pressure reached up to 14,500 psi and the working output pressure was 11,500 psi with a flow rate of 7.5 liters per minute. The crash chamber consists of a slurry injection line, a high pressure injection line and a sphere-shaped crash ball made of stainless steel. The slurry was directly injected into the face of the crash ball and the high pressure water was injected from the side of the slurry injection line. A schematic drawing is shown in Figure 2. The injected slurry and high pressure water were mixed at the bottom of the chamber and flown out to the solid/liquid separation part. Slurry was pressurized by the separation pump and flown into a hydrocyclone (Model: U2-gMax, Krebs). Solid and suspension were separated and the solid portion can be used as clean soil. 
There are two different contaminant removal mechanisms within this method, which are, physical desorption and chemical solubilization. High pressure water was used in this experiment to apply a sufficient physical impact force to the soil. Two kinds of high impact forces were applied to the contaminated soil. Initially, collision between the soil slurry and high pressure water occurred and secondly, collision between the accelerated soil slurry and metal crash ball occurred later. While the soil was subjected to physical impact force, two kinds of effects were expected. First, some of the soil-contaminant bindings were weakened or broken if the impact force was larger than the binding force. This means that the contaminant was desorbed from the soil particle and was dissolved or freely suspended in the surrounding liquid. Secondly, the formation of the micro-structural unit was broken and the soil particles in the aggregation were dispersed in all directions. Then the apparent specific surface area of the soil will be increased and the contaminants in the entrapped pores were exposed to the washing solution.

Removal efficiency can be enhanced with the addition of chemical solutions. EDTA is a well-known and widely-used chemical in soil washing for heavy metal contaminated soil. The contaminant removal efficiency of the chemical treatment in a conventional soil washing system for fine-grained soil is generally fairly low. In this new type of soil washing system, however, the efficiency of the chemical treatment for fine-grained soil can be enhanced by the effects of the physical impact force. In this case, a synergy effect between the physical and chemical reactions occurs and the removal efficiency can be enhanced.
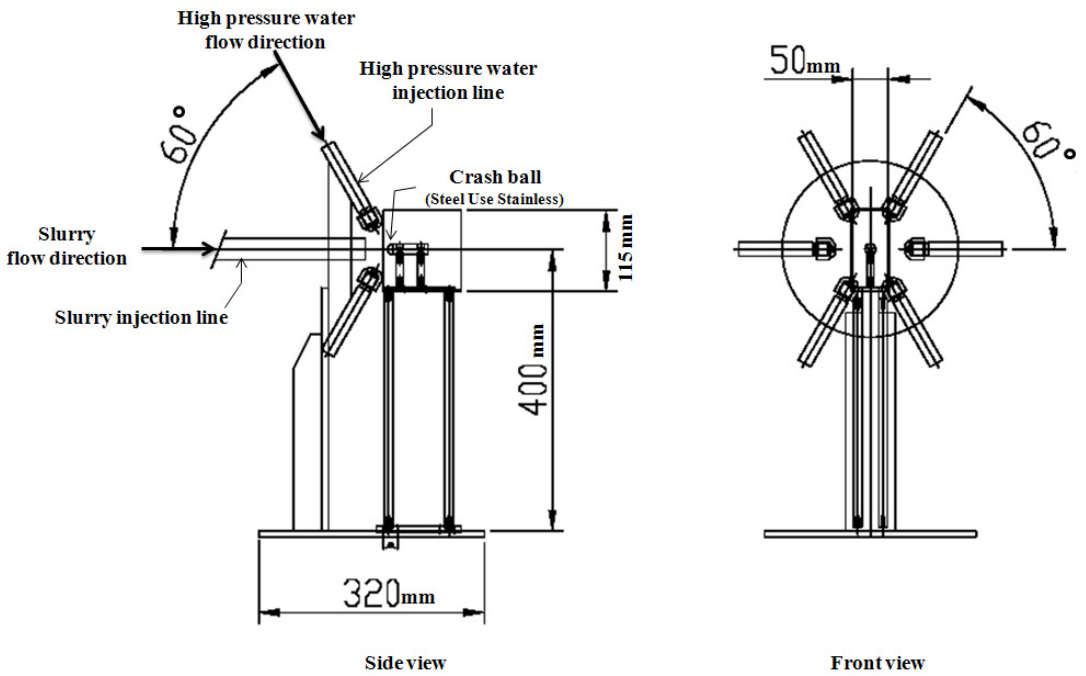

Figure 2: Schematic drawing of inside of crash chamber. 


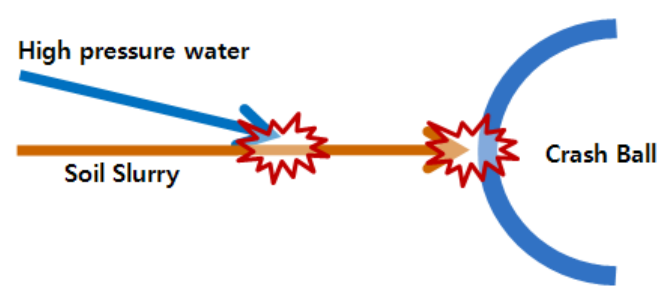

Figure 3: Schematic drawing of sequential physical impact on the contaminated soil.

\section{Experimental setup}

\subsection{Materials}

Soils taken from an old refinery site in South Korea were tested in this study. Surface soil (depth of $1 \mathrm{~m}$ ) was taken from the site followed by the measurement of the contaminant concentration of the original soil. The original soil was contaminated by various kinds of heavy metals; $\mathrm{Pb}, \mathrm{Cu}$ and As. For the soil washing tests, the soil was prepared by being sieved through a no. 10 mesh (2 $\mathrm{mm}$ ) and thoroughly homogenized. EDTA was used as a washing chemical solution.

\subsection{Method}

Three kinds of experiments were performed; physical desorption, chemical solubilization and a combination of the two. The same quantities of soil (12 kg) and tap water $(120 \mathrm{~L})$ were used for each test. The heavy metal concentration measurement for all test samples was performed at the National Instrumentation Center for Environmental Management (NICEM, Seoul National University, Korea) with the Korean Standard method.

\subsubsection{Test for physical desorption}

Contaminated soil and tap water were poured into the slurry reservoir and mixed for five minutes in order to spread the washing agent evenly in the slurry. After mixing, the slurry and high pressure water were injected into the crash chamber followed by solid/liquid separation. The soil sample was collected after separation.

\subsubsection{Test for chemical solubilization}

$0.05 \mathrm{mM}$ of EDTA was used as a washing agent. The contaminated soil and the EDTA solution were poured into the slurry reservoir. The slurry was mixed for five minutes, which is the sum of the homogeneity maintaining time and the equipment operation time in the physical forcing test method. After mixing, the tested slurry was collected and the soil was separated from the slurry using centrifugation. 


\subsubsection{Test for combined method}

The contaminated soil was mixed well for five minutes with the same washing solution as that which was used for the chemical reaction test. After mixing, the slurry and high pressure water were injected into the crash chamber, in the same way as the physical forcing method test. The soil sample was collected after the solid/liquid separation.

\section{Results and discussion}

The results are shown in Figure 4, which gives the heavy metal concentrations in $\mathrm{mg} / \mathrm{kg}$. Figure 5 shows the removal efficiency in \%. A similar trend was seen in each heavy metal experiment. In the case of the physical forcing method test, no chemical effect was considered since no reagents were mixed in with the slurry. From this approach, the removal efficiency is basically the result of physical desorption. In this paper, the detailed contaminant removal contribution of the physical impact was not investigated.

In the case of the chemical reaction test, physical desorption also occurred. At the time of mixing, from the mechanical stirring, the mechanism of physical attrition occurred and the removal efficiency includes both mechanisms.

In comparing results between the chemical reaction and combination method, the efficiencies were increased. These increases show the effect of using high pressure water. The difference between the two methods was the existence of a high pressure water system. As mentioned before, the chemical reaction could not occur sufficiently in fine-grained soil washing due to the existence of micro-structural units. In the case of the combination method, increased efficiency might be coming from the effect of the breakage of micro-structural units by high pressure impact. The most significant removal efficiency was found in the combination of physical and chemical treatments as $89 \%$ of $\mathrm{Pb}$, $87 \%$ of $\mathrm{Cu}$ and $84 \%$ of As were recovered.

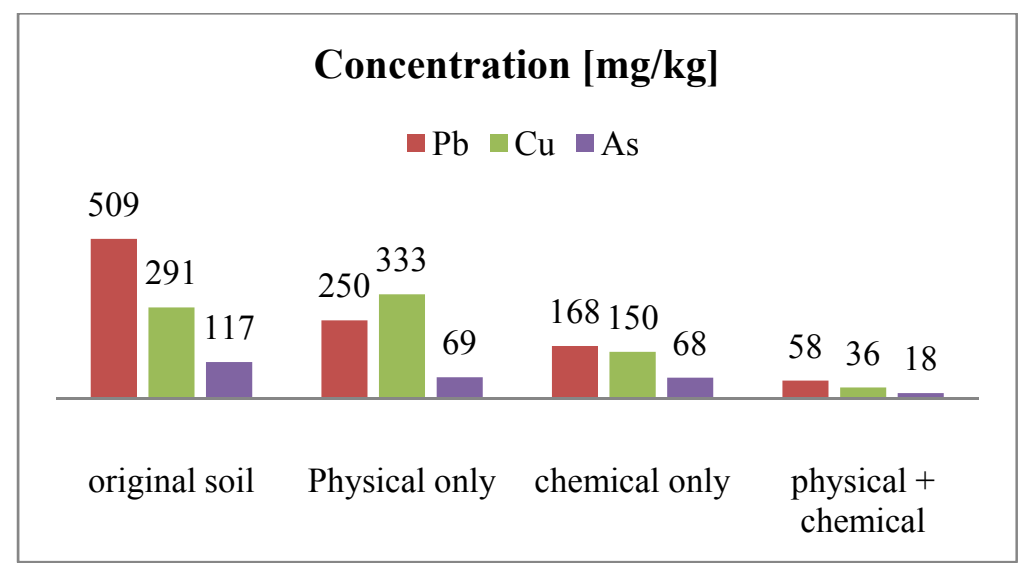

Figure 4: Concentration of original and treated soils. 


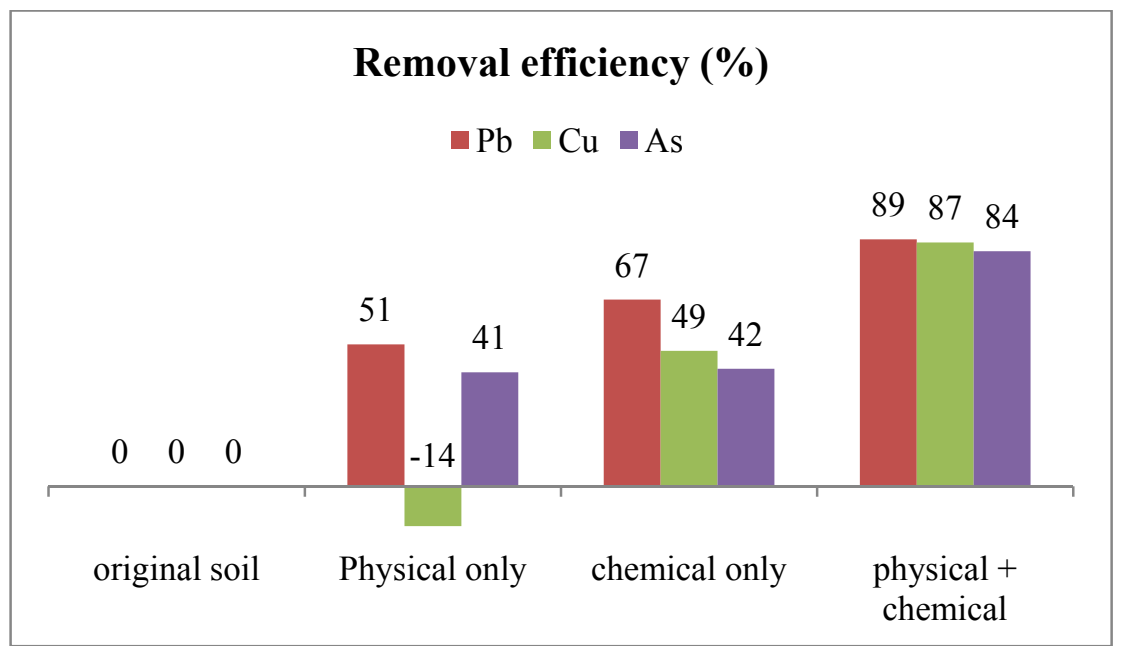

Figure 5: Removal efficiency of each treatment method.

\section{Summary and conclusion}

A novel soil washing methodology was developed and the evaluation for the pilot-scale equipment was performed in this study. Physical forces including direct impact force, mechanical attrition and the friction effect can attribute to the removal of contaminants from the soil. The efficiency of the chemical reaction in fine-grained soil washing can be enhanced by using a proper physical forcing system. The efficiency of the whole soil washing system can be enhanced by the optimization of the experimental conditions, with all the conditions considered.

\section{Acknowledgement}

This research was financially supported by The Republic of Korea Ministry of Environment as the "Green Remediation Research Center for Organic-Inorganic Combined Contamination".

\section{References}

[1] Chu, W., Remediation of contaminated soils by surfactant aided soil washing. Practice Periodical of Hazardous, Toxic, and Radioactive Waste Management, 7(1), pp. 19-24, 2003.

[2] Deshpande, S., Shiau, B. J., Wade, D., Sabatini, D. A. \& Harwell, J. H., Surfactant selection for enhancing ex situ soil washing. Water Research, 33(2), pp. 351-360, 1999. 
[3] Feng, D., Lorenzen, L., Aldrich, C. \& Mare, P. W., Ex situ diesel contaminated soil washing with mechanical methods. Minerals Engineering, 14(9), pp. 1093-1100, 2001.

[4] Khalladi, R., Benhabiles, O., Bentahar, F. \& Mou-lai-Mostefa, N., Surfactant remediation of diesel fuel polluted soil. Journal of Hazardous Materials, 164, pp. 1179-1184, 2009.

[5] Kuhlman, M. I. \& Greenfield, T. M., Simplified soil washing processes for a variety of soils. Journal of Hazardous Materials, 66, pp. 31-45, 1999.

[6] Mann, M. J., Full-scale and pilot-scale soil washing. Journal of Hazardous Materials, 66, pp. 119-136, 1999.

[7] Paria, S., Surfactant enhanced remediation of organic contaminated soil and water. Advances in Colloid and Interface Science, 138, pp. 24-58, 2008.

[8] Yong, R. N. \& Mulligan, C. N., The impact of clay microstructural features on the natural attenuation of contaminants. Applied Clay Science, 23, pp. 179-186, 2003. 